\title{
Cervical Sympathetic Chain Schwannoma: A Case Report
}

\author{
S. K. Awasthi · Angshuman Dutta
}

Received: 18 May 2011/Accepted: 24 May 2011/Published online: 8 June 2011

(C) Association of Otolaryngologists of India 2011

\begin{abstract}
Tumors originating in the parapharyngeal space are rare and are fascinating to the surgeon in view of the anatomical complexity of the area, varied histological type of tumors encountered and the impressive size which some of these tumors attain before becoming clinically evident. We report a rare case of schwannoma arising from the cervical sympathetic chain presenting as a mass in the parapharyngeal space.
\end{abstract}

Keywords Schwannoma $\cdot$ Parapharyngeal space

\section{Introduction}

Parapharyngeal space tumors are rare and account for $0.5 \%$ of all head and neck cancers [1]. Among the parapharyngeal space tumors approximately $50 \%$ are salivary gland in origin and $20 \%$ are neurogenic [2]. The most common neurogenic tumor found in the parapharyngeal space is schwannoma [2]. We report a rare case of schwannoma arising from the cervical sympathetic chain presenting as a mass in the parapharyngeal space.

\section{Case Report}

40 year old male presented with complaints of odynophagia for 2 months. There was no history of any lump neck,

S. K. Awasthi

Department of ENT, Command Hospital (AF), Bangalore, India

A. Dutta $(\bowtie)$

Department of ENT, 5 Air Force Hospital, Jorhat, India

e-mail: debdas.debmita@rediffmail.com hoarseness, nasal regurgitation, sore throat, otalgia or weight loss. Examination revealed a visible bulge of the right tonsil and right posterior pillar (Fig. 1). Neck examination showed a diffuse bulge below angle of right mandible. There were no palpable neck nodes. Indirect laryngoscopy showed a bulge of right lateral pharyngeal wall with epiglottis being pushed slightly to the left. However both vocal cords were mobile. Cranial nerve examination was normal. A provisional diagnosis of Right parapharyngeal mass was made. Contrast enhanced CT scan of the neck showed a $6 \mathrm{~cm} \times 5 \mathrm{~cm} \times 3.6 \mathrm{~cm}$ size circumscribed mass occupying right parapharyngeal space with peripheral rim enhancement (Fig. 2). There was no involvement of neck vessels and there were no neck nodes. FNAC of the mass was suggestive of a schwannoma. Patient was taken up for excision of the mass through a transcervical approach. Common facial vein and facial artery were ligated and submandibular gland retracted up. Right sternocleidomastoid was retracted and carotid sheath was opened. The tumor was found to be arising from the cervical sympathetic chain just above and posterior to the bifurcation of carotid artery (Fig. 3). The internal jugular vein was found displaced laterally and right vagus was found to be intact. The tumor was found to be well encapsulated and by blunt dissection the superior and inferior aspect of the tumor was identified and the tumor was excised (Fig. 4) along with an intervening segment of the nerve. Post operatively he was found to have developed Horners' syndrome however patient himself was asymptomatic. Grossly the excised tumor measured $6 \mathrm{~cm} \times 5 \mathrm{~cm} \times 4.5 \mathrm{~cm}$. Microscopic sections of the tumor areas revealed sheets of spindle shaped cells (nerve cells) (Fig. 5) which were at places forming palisaded nuclei (Verocay bodies). Both cellular (Antoni A) and hypocellular, loose and degenerative (Antoni B) areas were seen. Impression was of a schwannoma. 


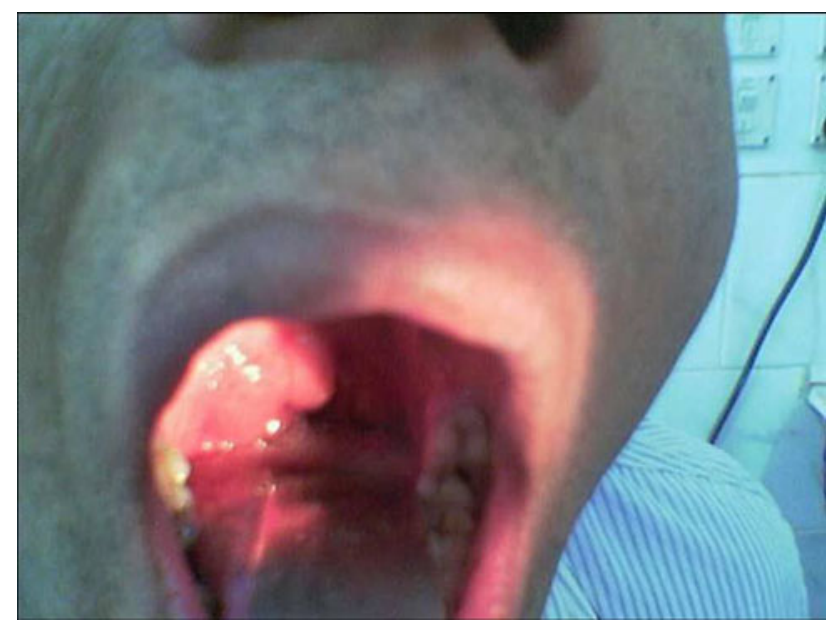

Fig. 1 Preoperative photograph of the patient showing bulge of the right tonsil

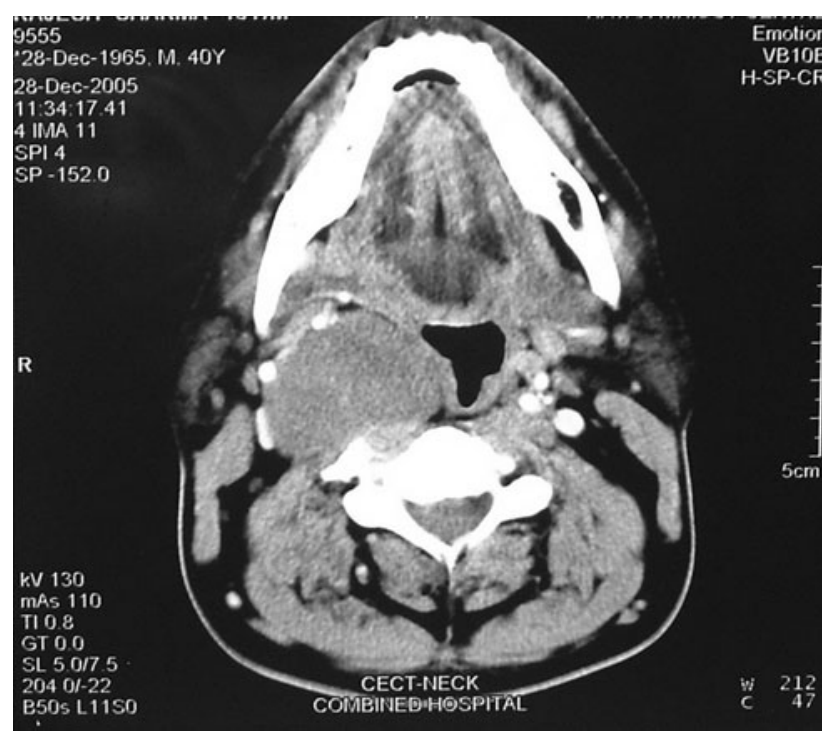

Fig. 2 CECT neck showing the parapharyngeal mass occuping the poststyloid compartment

\section{Discussion}

Schwannomas are benign solitary nerve sheath tumors arising from any nerve with Schwann cells except olfactory and optic which lack them [3, 4]. In the head and neck region they arise most commonly from the parapharyngeal space [3]. Most parapharyngeal neurogenic tumors are schwannomas arising from the vagus and less commonly from the sympathetic chain or hypoglossal [5]. Majority of schwannomas are asymptomatic at time of presentation and may have non specific symptoms such as dysphagia or sore throat. The presentation is by exerting pressure on the surrounding structures. Expansion of tumor occurs in the plane of least resistance medially towards the tonsil and

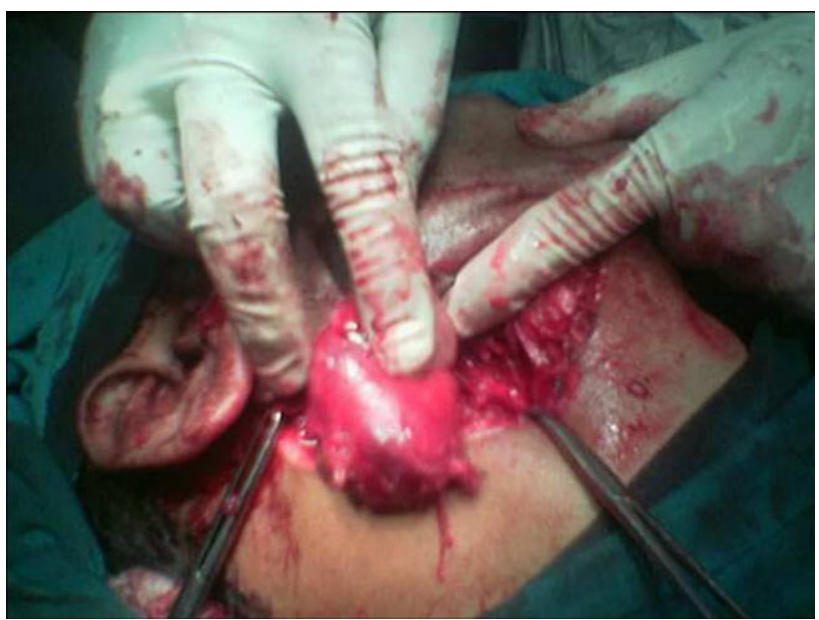

Fig. 3 Intraoperative photograph showing the tumor being exposed

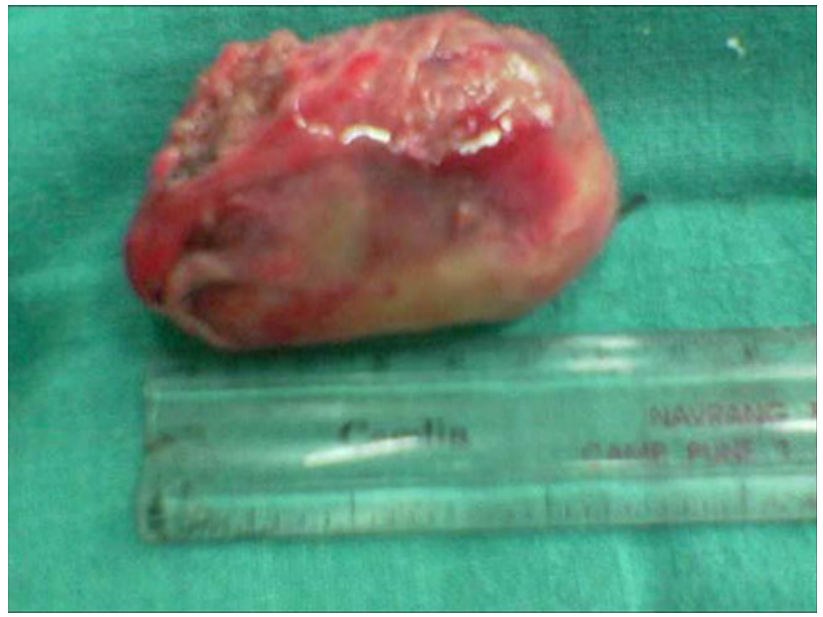

Fig. 4 Figure showing the excised specimen

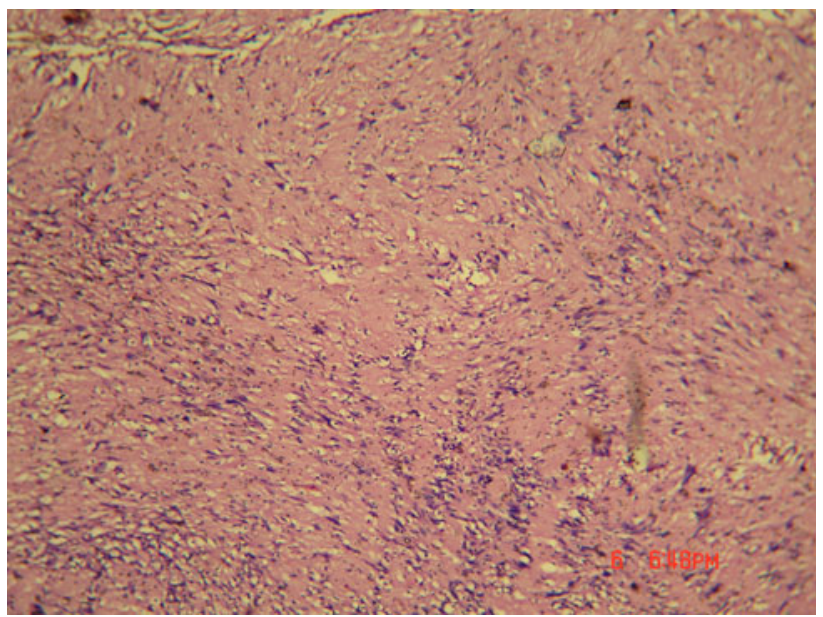

Fig. 5 Microscopic sections (low power) of the tumor showing sheets of spindle shaped cells (nerve cells) 
lateral pharyngeal wall and posteriorly into retromandibular area presenting as a medial displacement of tonsil, soft palate and pharynx or presenting as a mass near angle of mandible [1, 2]. Paralysis of the nerve is rare but as the tumor grows they can obstructive symptoms and loss of nerve function $[1,2]$.

Neurogenic tumors of head and neck arise from neural crest cells which differentiate into nerve sheath cells and sympathoblast. Schwann cell is the parent cell of both schwannoma and neurofibroma. Neurofibroma also has an origin from the perineurium and is thus linked inseparably from the nerve of origin [5] and cannot be separated from it. Schwannomas grow longitudinally along the length of the nerve assuming a fusiform appearance without compromising the morphological and functional integrity of the nerve and can be separated surgically from their nerve of origin. Distinction between schwannoma and neurofibroma can be made only microscopically. Neurofibromatosis may be multiple as a part of Von Recklinghausen's syndrome (NF1) or rarely may be single. Schwannomas are usually solitary and may rarely be malignant.

Most important aspect of these lesions is distinguishing cervical schwannoma from other pathology like salivary gland tumors and paragangliomas in the parapharyngeal space. The differential diagnosis of a parapharyngeal mass is based on the division of the space into prestyloid and poststyloid compartments [3]. Contrast enhanced CT scan is the best initial diagnostic study to determine the size and extent of tumor, possible origin of tumor based on displacement of carotid sheath and preservation of parapharyngeal fat and to demonstrate degree of tumor vascularity. CT scan also helps plan the surgical approach and to counsel patient preoperatively about postoperative neurological deficiency. FNAC has revolutionized the ability of clinician to diagnose masses present in the head and neck area. However ability of FNAC to reveal diagnosis is poorly defined in neurogenic tumors [6]. In a study by Colreavy et al. in neurogenic tumors [4] FNAC was found to give an accuracy of only $25 \%$.
Schwannomas being well encapsulated tumors can be completely removed during surgery. Selection of the approach to the parapharyngeal space is based on the site and size of the tumor keeping in mind adequate exposure of the tumor and exposure of the neurovascular structures in the vicinity to preserve their function and control bleeding $[1,2]$. Transcervical, transmandibular and transparotid are the main approaches described [1] to approach the parapharyngeal space. Postoperative complications involved removing the cervical sympathetic chain involve Horner's syndrome with miosis, anhidrosis, enopthalmos and ptosis but is generally not noticed by the patient. In a study by Mark et al. [7] of 4 cases of cervical schwannoma operated the nerve could not be dissected free from the tumor and had to be sacrificed. In our case the cervical sympathetic chain could not be dissected free from the tumor and a segment of nerve was sacrificed and patient developed postoperative Horners' syndrome.

\section{References}

1. Pang KP, Goh CHK, Tan HM (2002) Parapharyngeal tumors: an 18 yr review. J Laryngol Otol 116:170-175

2. Olsen KD (1994) Tumors and surgery of the parapharyngeal space. Laryngoscope 104:1-28

3. Mikaelian DO, Holmes WF, Simonian SK (1981) Parapharyngeal schwannomas. Otolaryngol Head Neck Surg 89:77-81

4. Colreavy MP, Lacy PD, Hughes J, Bouchier-Hayes D, Brennan P, O’Dwyer AJ (2000) Head and neck schwannomas a 10 yr review. J Laryngol Otol 114:119-124

5. Watkinson JC, Gaze MN, Wilson JA (2000) Benign neck disease. In: Stell and Maran's head and neck surgery, 4th edn. Butterworth Heinemann, Oxford, pp 193-194

6. Ganesan S, Harar RPS, Owen RA et al (1997) Horner's syndrome: a rare presentation of cervical sympathetic chain schwannoma. J Laryngol Otol 111:493-495

7. Mark KW, Samuel SG, Robinson JL, Weissmann JL (2004) Cervical sympathetic chain schwannoma. Laryngoscope 114(12): 2210-2213 